\title{
Bloch particle in presence of $d c$ and ac fields: Statistics of the Wigner delay time
}

\author{
M. Glück, A. R. Kolovsky* and H. J. Korsch \\ Fachbereich Physik, Universität Kaiserslautern, D-67653 Kaiserslautern, Germany
}

(November 17, 1998)

\begin{abstract}
The paper studies quantum states of a Bloch particle in presence of external ac and dc fields. Provided the period of the ac field and the Bloch period are commensurate, an effective scattering matrix is introduced, the complex poles of which are the system quasienergy spectrum. The statistics of the resonance width and the Wigner delay time shows a close relation of the problem to random matrix theory of chaotic scattering.
\end{abstract}

PACS: 03.65.†, 05.45.+b, 73.20Dx

This paper studies quantum states of a Bloch particle in presence of external ac and dc fields

$$
\widehat{H}=\hat{p}^{2} / 2+\cos x+F x+F_{\omega} x \cos (\omega t) .
$$

Although being of very physical importance, this problem proves to be pretty tough and was analyzed before exclusively in a one-band approximation by using the tightbinding model [1]. In our recent paper [2] we have suggested a method which allows us to go beyond a $n$-band approximation. For the case of a one to one resonance between the Bloch period $T_{B}=\hbar / F$ and the period of ac field $T_{\omega}=2 \pi / \omega$ the analysis of the complex quasienergy spectrum was done in Ref. [3]. It was shown that in the generic case, where the classical dynamics of the system (1) (with the static field canceled) is chaotic [5], the spectrum should be regarded as quasi-random. Then the statistical analysis is an appropriate one for describing the spectrum. The distribution of decay times of the quantum metastable states (the decay time is defined by the imaginary part of complex quasienergy) was found to have an algebraic tail, which is responsible for a specific phenomenon of quantum stabilisation reported in Ref. [3,4].

In this present letter we analyze the model (1) under a weaker condition of commensurate periods: $T_{B} / T_{\omega}=r / q$ $(r, q$ are coprime integers). We show that in this case the eigenvalue problem for the Hamiltonian (1) can be reduced to an effective scattering problem with $q$ open channels. In other words, we introduce an effective scattering matrix of size $q \times q$, the complex poles of which are the complex resonance spectrum. This is a central result of the paper [6]. Further analysis involves the famous conjecture in the theory of quantum chaos that, provided the classical dynamics of the system is chaotic, the system spectral properties are similar to those of a random matrix sharing the same symmetry [7]. Using the results of modern random matrix theory of chaotic scattering (see [8] and references therein) we can predict the asymptotic behavior of the distributions for the decay and delay times. In particular, it follows that in the incommensurate case (which can be approached through the limit $r, q \rightarrow \infty, r / q \rightarrow$ irrational) the distribution has no algebraic tail. This explains the absence of quantum stabilisation in the incommensurate case observed in the numerical simulation of the system dynamics $[3,4]$.

We briefly recall some results of the papers $[2,3]$. It is convenient to include the ac term of the Hamiltonian (1) in the periodic potential, which is done by the gauge transformation $p \rightarrow p+\left(F_{\omega} / \omega\right) \sin (\omega t), x \rightarrow$ $x-\left(F_{\omega} / \omega^{2}\right) \cos (\omega t)$. Then the Hamiltonian takes the form

$$
\begin{gathered}
\widehat{H}=\hat{p}^{2} / 2+V(x, t)+F x, \\
V(x, t)=\cos [x-\epsilon \cos (\omega t)], \quad \epsilon=F_{\omega} / \omega^{2} .
\end{gathered}
$$

The dynamics of the system (2) is determined by the system evolution operator which, provided the existence of the common period $T=q T_{B}=r T_{\omega}$, is periodic in time [2]. Thus time-Floquet formalism can be used and, denoting by $\widehat{U}$ the Floquet operator (i.e., the evolution operator over the period $T$ ), the equation for the system quasienergies has the form

$$
\widehat{U} \psi(x)=\exp (-i \lambda) \psi(x) .
$$

Equation (3) should be accomplished by a boundary condition, the type of which defines whether the spectrum is complex (and discrete) or real (and continuous). In this paper we consider a hermitian boundary condition. Thus $\lambda$ is real in what follows and the eigenfunctions are normalized against $\delta$-function.

We obtain a constructive analytical expression for the unitary operator $\widehat{U}$ by using the standard substitution $\psi(x, t)=\exp (-i F x t / \hbar) \tilde{\psi}(x, t)$ in the Schrödinger equation. Then

$$
\begin{gathered}
\widehat{U}=e^{-i q x} \widehat{W}, \\
\widehat{W}=\widehat{\exp }\left\{-\frac{i}{\hbar} \int_{0}^{T}\left[\frac{(\hat{p}-F t)^{2}}{2}+V(x, t)\right] \mathrm{d} t\right\},
\end{gathered}
$$

where the caret over exponent denotes time ordering. It is seen from Eq. (4) that the operator $\widehat{U}$ commutes with the translational operator over the lattice period and, therefore, the quasimomentum $k$ is a good quantum number. Presenting the wave function in Eq. (3) in the form 


$$
\psi(x)=e^{i k x} \sum_{n=-\infty}^{\infty} c_{n}^{(k, \lambda)}\langle x \mid n\rangle, \quad\langle x \mid n\rangle=(2 \pi)^{-1 / 2} e^{i n x}
$$

we reduce the eigenvalue problem (3) to diagonalisation of an infinite matrix given by the product of two unitary matrices:

$$
S W \mathbf{c}=\exp (-i \lambda) \mathbf{c}
$$

In Eq. (6), $S$ is the shift matrix with the elements $S_{n^{\prime}, n}=\left\langle n^{\prime}|\exp (-i q x)| n\right\rangle=\delta_{n^{\prime}, n-q}$ and the elements of the matrix $W$ are given by $W_{n^{\prime}, n}=$ $\left\langle n^{\prime}|\exp (-i k x) W \exp (i k x)| n\right\rangle$ (note that the quasimomentum $k$ enters as a parameter). By convention the expansion coefficients $c_{n}^{(k, \lambda)}$ are arranged in a column vector $\mathbf{c}$ with an index $n$ decreasing from up to down and, to simplify the formulas, we shall omit the quasimomentum index $k$ and quasienergy index $\lambda$.

First we consider the case $q=1$. The method of solving Eq. (6) is based on the fact that the matrix $W$ tends asymptotically to a diagonal one

$$
\begin{gathered}
W_{n^{\prime}, n} \rightarrow \delta_{n^{\prime}, n} w_{n}, \quad n, n^{\prime} \rightarrow \pm \infty, \\
w_{n}=\exp \left[-\frac{i}{2 \hbar} \int_{0}^{T}(\hbar n+\hbar k-F t)^{2} \mathrm{~d} t\right] .
\end{gathered}
$$

Let us assume that the asymptotic (7) is "good enough" for $|n|>N$. [The characteristic size of the "interaction region", where the asymptotic fails, is given by $2 N^{*} \approx$ $J / 2 \pi \hbar$ with $J$ being the volume of the chaotic component of the classical phase space (see Fig. 1 in Ref. [3]). In the regular case $\epsilon=0, J$ is the volume under separatrix.] Then we decompose the vector $\mathbf{c}$ into three sub-vectors

$$
\mathbf{c}=\left(\begin{array}{l}
\mathbf{c}^{(+)} \\
\mathbf{c}^{(0)} \\
\mathbf{c}^{(-)}
\end{array}\right)
$$

where $\mathbf{c}^{(+)}$consists of the coefficients $c_{n}$ with indices $n>$ $N$, $\mathbf{c}^{(-)}-$with $n<-N-1$, and $\mathbf{c}^{(0)}$ is constructed from $c_{n}$ with indices $-N-1 \leq n \leq N$. The vector $\mathbf{c}^{(+)}$is completely defined by the value of the coefficient $c_{N+1}$ and the equation

$$
w_{n} c_{n}=\exp (-i \lambda) c_{n-1},
$$

which follows from Eq. (6) for $q=1$ and asymptotic (7). Analogously, the vector $\mathbf{c}^{(-)}$is defined by Eq. (9) and the value of $c_{-N-1}$. For the vector $\mathbf{c}^{(0)}$ we have an algebraic equation

$$
\left[\widehat{Q}_{N}(S W)-\exp (-i \lambda)\right] \mathbf{c}^{(0)}=-w_{N+1} c_{N+1} \mathbf{e}_{N}
$$

In Eq. (10) $\widehat{Q}_{N}(S W)$ is the truncated matrix $S W$

$$
\widehat{Q}_{N}(S W)=\left(\begin{array}{ll}
0_{1 \times(2 N+1)} & 0_{1 \times 1} \\
W_{(2 N+1) \times(2 N+1)} & 0_{(2 N+1) \times 1}
\end{array}\right)
$$

and $\mathbf{e}_{N}$ is a column vector of the same size $2 N+2$ with all elements equal to zero except the first one, equal to unit. We note that Eq. (10) actually relates the coefficient $c_{-N-1}$ to the coefficient $c_{N+1}$ and, thus, matches two asymptotic solutions $\mathbf{c}^{(+)}$and $\mathbf{c}^{(-)}$. Without loss of generality we can choose the phase of $c_{N+1}$ such that $-w_{N+1} c_{N+1}=1$.

We define the matrix $G(\lambda)$ (of dimension $1 \times 1$ in the considered case $q=1$ ) as a phase gain (loss) relative to the case when the matrix $W$ is given by Eq. (7) for arbitrary $n, n^{\prime}$ (we shall refer to the latter case as "zero solution"). Thus

$$
c_{-N-1}=G(\lambda) \tilde{c}_{-N-1}
$$

where $\tilde{c}_{-N-1} \sim \exp [i(2 N+2) \lambda]$ is the zero solution. Using Eq. (10), the matrix $G(\lambda)$ can be presented in the form

$$
G(\lambda)=\lim _{N \rightarrow \infty} a_{N}(\lambda) \mathbf{e}_{N}^{\prime}\left[\widehat{Q}_{N}(S W)-\exp (-i \lambda)\right]^{-1} \mathbf{e}_{N} .
$$

where $\mathbf{e}_{N}^{\prime}$ is a row vector with all elements equal to zero except the last one, equal to unit, and the phase factor $a_{N}(\lambda)=\tilde{c}_{-N-1}^{*}$ is given by the zero solution. We also add the limit $N \rightarrow \infty$ in the Eq. (13) which insures the validity of asymptotic formula (7). The numerical calculation of the scattering matrix (13) indicates a rapid convergence of the limit.

An important characteristic of $G(\lambda)$ is the quantity

$$
\tau=-i \frac{\mathrm{d} \ln G(\lambda)}{\mathrm{d} \lambda}=-i G^{*} \frac{\mathrm{d} G}{\mathrm{~d} \lambda}
$$

which is known in the literature as the Wigner delay time (note, that here $\tau$ can take negative values) and is directly related to density of states of a continuous quasienergy spectrum. The quantity $\tau$ also determines the normalization of the sub-vector $\mathbf{c}^{(0)}$. [The whole vector $\mathbf{c}_{\lambda}$ is normalized against a $\delta$-function: $\left\langle\mathbf{c}_{\lambda^{\prime}} \mid \mathbf{c}_{\lambda}\right\rangle=\delta\left(\lambda^{\prime}-\lambda\right)$.] Namely,

$$
\tau=\lim _{N \rightarrow \infty} \sum_{n=-N-1}^{N}\left(\left|c_{n}^{(0)}\right|^{2}-1\right) .
$$

Calculation of the delay time $\tau$ on the basis of Eq. (15) is actually preferable compared to Eq. (14) because it eliminates a numerical estimation for the derivative.

As an example, the left panel in Fig. 1 shows the delay time $\tau$ as a function of the quasienergy $\lambda$ and quasimomentum $k$ for the parameters $\omega=10 / 6, \epsilon=F_{\omega} / \omega^{2}=$ $1.5, \hbar=0.5$, and $F=\hbar / T_{\omega} \approx 0.13$ of the Hamiltonian (2). In addition, the right panel in Fig. 1 (after [3]) depicts the real part of the complex quasienergy corresponding to the most stable states [9]. As expected, the 
quantity $\tau=\tau(\lambda, k)$ reveals the underlying resonance structure.

In this paragraph we generalize the main formulas displayed above for the case of arbitrary $q$. For arbitrary $q$, Eq. (9) has the form

$$
w_{n} c_{n}=\exp (-i \lambda) c_{n-q} .
$$

It follows from Eq. (16) that there are $q$ independent solutions $\mathbf{c}^{( \pm, i)},(i=1, \ldots, q)$, and, therefore, the matrix $G(\lambda)$ is of dimension $q \times q$. We adopt Eq. (13) for this case by substituting the vector $\mathbf{e}_{N}$ by the matrix $e_{N}$ of the size $q \times(2 N+1+q)$ of the following structure (shown for $q=2, N=2$ )

$$
e_{N}=\left(\begin{array}{ll}
1 & 0 \\
0 & 1 \\
0 & 0 \\
0 & 0 \\
0 & 0 \\
0 & 0 \\
0 & 0
\end{array}\right), \quad e_{N}^{\prime}=\left(\begin{array}{lllllll}
0 & 0 & 0 & 0 & 0 & 1 & 0 \\
0 & 0 & 0 & 0 & 0 & 0 & 1
\end{array}\right)
$$

and the prefactor $a_{N}$ by the diagonal $q \times q$ matrix with the elements given by the zero solutions. The quantity Eq. (14) is also a matrix $\tau=-i G^{+} \mathrm{d} G / \mathrm{d} \lambda$ and the normalization condition Eq. (15) takes the form $\tau_{i, i}=\lim _{N \rightarrow \infty} \sum_{n=-N-q}^{N}\left(\left|c_{n}^{(0, i)}\right|^{2}-1 / q\right)$.

The rest of the paper is devoted to the statistics of the delay and decay times. First we discuss which result could be expected. To avoid a misunderstanding, we also recall that the decay and delay times are related but not identical notions. The decay time is the life-time of a metastable states and is defined as $\tau^{\prime}=1 / \Gamma$, where $\Gamma$ is the resonance width, i.e., twice the imaginary part of the complex (quasi-)energy. Thus the decay time is a characteristic of the complex spectrum. In contrast, the delay time Eq. (14) is a characteristic of continuous real spectrum. Physically it corresponds to the time interval, during which a wave packet (coming from the asymptotic region) is captured within the interaction region.

Statistical approach to chaotic scattering, which is based on the random matrix assumption (the system Hamiltonian is modeled by a random matrix), is nowadays widely used in the problems like ballistic electron scattering in the mesoscopic devices, decay of unstable nuclei and molecules, and many others [10]. In these cases the general theory predicts the following distributions of the normalized partial Wigner delay time $\tau$ and resonance width $\Gamma$ (inverse decay time) [11]

$$
\begin{gathered}
P(\tau)=\frac{1}{M !} \frac{1}{\tau^{M+2}} \exp \left(-\frac{1}{\tau}\right), \\
\Pi(\Gamma)=\frac{M^{M}}{(M-1) !} \Gamma^{M-1} \frac{d^{M}}{d \Gamma^{M}}\left(e^{-\Gamma} \frac{\sinh \Gamma}{\Gamma}\right),
\end{gathered}
$$

where $M$ is the number of open channels and the system is assumed to belong to GUE symmetry class [12]. A remarkable feature of the distributions displayed is the presence of an algebraic tail for both delay and decay times. (Note, however, that in the limit of an infinite number of channels the tail is absent.) Of course, we can not directly apply the formulas (18), (19) to our system of interest [13]. However, relaying on the universality of chaos, it can be used as "the first approximation".

The histogram in Fig. 2 shows the distribution of the Wigner delay time for the parameters of Fig. 1. It is seen that the peak of the distribution is around zero delay time and then the function slowly decays. The asymptotic of the distribution for large $\tau$ is presented in the insert of the figure, where we plot the integrated distribution $I(\gamma)=\int_{0}^{\gamma} \tilde{P}\left(\gamma^{\prime}\right) \mathrm{d} \gamma^{\prime}$ of the inverse delay time $\gamma=1 / \tau$ in double logarithmic scale. The integrated distribution of the resonance width $\Gamma$ is also shown. It follows from the numerical data that $I(\gamma) \sim \gamma^{2}, \gamma \ll 1$, and $I(\Gamma) \sim$ $\Gamma, \Gamma \ll 1$. Thus $P(\tau) \sim \tau^{-3}, \tau \gg 1$, in agreement with Eq. (18) for $M=1$, and $\Pi(\Gamma) \rightarrow$ const, $\Gamma \rightarrow 0$, in agreement with Eq. (19). A more detailed statistical analysis of the delay time, which includes the case $q \neq 1$ and the case of recovered orthogonal symmetry, will be published elsewhere.

To summarize, we rigorously (no approximations) analyzed the problem of a Bloch particle in ac and dc fields. The analysis is based on a scattering matrix approach, which is essentially the familiar scattering theory, however in momentum space. As the main result, we introduced an effective scattering matrix, which contains exhaustive information about the system (quasi-)energy spectrum. In addition and as an example of the application of the theory, we analyzed the statistics of the Wigner delay time for the system parameters, where its classical dynamics is chaotic. The obtained results are shown to be consistent with the predictions of random matrix theory of chaotic scattering. In this connection we would like to note that the system discussed suggests to be a nice physical model for testing the predictions of the random matrix theory - it is not easy to find other physical system, where the calculation of the resonances and delay time is so simple and without involving any additional assumption.

This research has been supported by the Deutsche Forschungsgemeinschaft (SPP "Zeitabhängige Phänomene und Methoden in Quantensystemen der Physik und Chemie" and GK "Laser und Teilchenspektroskopie").

* Also at L. V. Kirensky Institute of Physics, 660036 Krasnoyarsk, Russia.

[1] D. H. Dunlap and V. M. Kenkre, Phys. Rev. B 34, 3625 (1986); Nguyen Hong Shon and H. N. Nazareno, J. Phys.: Condens Matter 4, L611 (1992); X.-G. Zhao, R. Jahnke, 
and Q. Niu, Phys. Lett. A 202, 297 (1995).

[2] M. Glück, A. R. Kolovsky, H.-J. Korsch, and N. Moiseyev, EPJ (part D), in press.

[3] M. Glück, A. R. Kolovsky, and H.-J. Korsch, Phys. Lett. $\mathrm{A}$, in press.

[4] M. Glück, A. R. Kolovsky, and H.-J. Korsch, Phys. Rev. E, 58, 6835 (1998).

[5] The phase space of the system (1) for $F=0$ contains the chaotic component for almost all values of the parameters $\omega$ and $F_{\omega}$. Exclusions are the limits $F_{\omega} \rightarrow 0, \omega=$ const, and $F_{\omega}=$ const, $\omega \rightarrow \infty$.

[6] Let us note that the theory also covers the case of pure static field. In this case the complex poles of the effective scattering matrix are the famous Wannier ladder of resonances.

[7] Chaos and Quantum Physics, Proceedings of the LesHouches summer school, edited by M. J. Giannoni et. al. (North-Holland, Amsterdam, 1991).

[8] Yan V. Fyodorov and H.-J. Sommers, J. Math. Phys. 38, 1918 (1997).

[9] In Ref. [3] the metastable state of the system were obtained by solving the eigenvalue problem (6) with nonhermitian resonance-like boundary condition $\lim _{n \rightarrow+\infty}\left|c_{n}\right| \rightarrow 0, F>0$. (Should be opposed to the presently considered case of the hermitian boundary condition $\lim _{n \rightarrow \pm \infty}\left|c_{n}\right| \rightarrow 1$.)

[10] H. A. Weidenmüller, in Chaos and Quantum chaos, edited by W. D. Heiss (Springer, New York, 1992).

[11] See Eqs. (102), (162) in Ref. [8] for the case of perfect coupling, where the distributions have the most simple form.

[12] GUE symmetry class implies no time reversal symmetry. This is the case for a Bloch particle with an arbitrary quasimomentum $k$, excluding $k$ belonging to the center and edge of the Brillouin zone.

[13] In our forthcoming paper we develop an alternative random matrix approach based on CUE (Circular Unitary Ensemble) instead of GUE (Gaussian Unitary Ensemble). This approach will be more appropriate for the problem under study. In fact, the Hamiltonian (2) definitely can not be modeled by a random matrix and it is only the evolution operator (4), which can be considered as random in some sense.
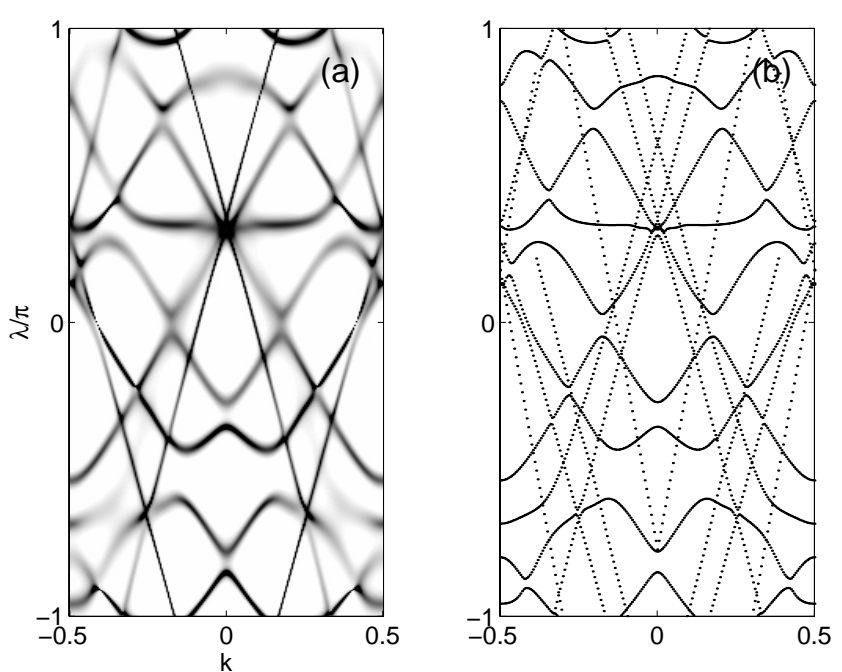

FIG. 1. Wigner delay time as a gray scale map (a) and the real part of the complex quasienergy spectrum (b) [for each $k$ first 13 most stable states is plotted]. The system parameters are $\omega=10 / 6, \epsilon=F_{\omega} / \omega^{2}=1.5, \hbar=0.5$, and $F=\hbar / T_{\omega} \approx 0.13$

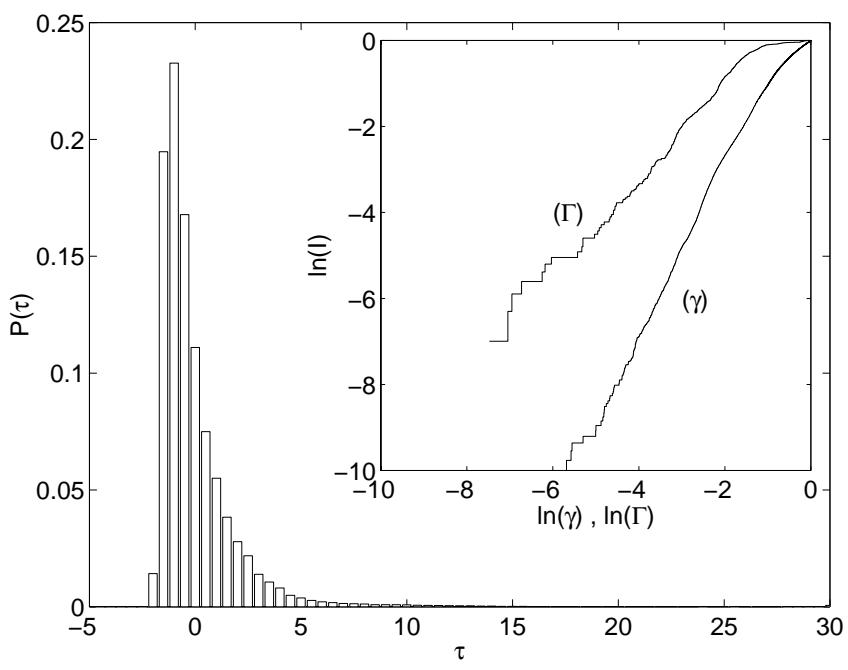

FIG. 2. The distribution of the Wigner delay time. The insert shows integrated distributions of inverse delay time $\gamma=1 / \tau$ and resonance width $\Gamma$. 